\title{
LOFAR status and first astronomical results
}

\section{R. C. Vermeulen ${ }^{1}$ on behalf of the LOFAR collaboration}

ASTRON Netherlands Institute for Radio Astronomy

P.O. Box 2, 7990 AA Dwingeloo, The Netherlands

E-mail: rvermeuleneastron.nI

LOFAR, the Low Frequency Array, is a next-generation radio telescope designed by ASTRON, with antenna stations concentrated in the north of the Netherlands and currently spread into Germany, France, Sweden and the United Kingdom; plans for more LOFAR stations exist in several other countries. Utilizing a novel, phased-array design, LOFAR is optimized for the largely unexplored low frequency range between 30 and $240 \mathrm{MHz}$. Digital beam-forming techniques make the LOFAR system agile and allow for rapid re-pointing of the telescopes as well as the potential for multiple simultaneous observations. Processing (e.g. cross-correlation) takes place in the LOFAR BlueGene/P supercomputer and associated post-processing facilities. With its dense core (inner few km wide) array and long (more than $1000 \mathrm{~km}$ ) interferometric baselines, LOFAR reaches unparalleled sensitivity and resolution in the low frequency radio regime. The International LOFAR Telescope (ILT) is currently reviewing proposals that will be selected for observing in the first fully operational cycle, starting in December 2012. Part of the allocations will be made on the basis of a fully Open Skies policy; there are also reserved fractions assigned by national consortia in return for contributions from their country to the ILT. In this invited talk, the gradually expanding complement of operationally verified observing modes and capabilities are reviewed, and some of the exciting first astronomical results from the commissioning period are presented.

11th European VLBI Network Symposium \& Users Meeting

October 9-12, 2012

Bordeaux, France

\footnotetext{
${ }^{1}$ Speaker
} 


\section{Introduction, technical design}

\subsection{Dipole receptors and phased-array beam-forming}

LOFAR, the Low Frequency Array, was designed and built by ASTRON for innovative science and in-depth exploration of the spectral window below $240 \mathrm{MHz}$. The International LOFAR Telescope (ILT) is an array of dipole antenna stations distributed across parts of the Netherlands and into other European countries with central processing facilities for interferometry and other applications. The stations have no moving parts: dipole low-band antennas (LBA) function down to $10 \mathrm{MHz}$ and are optimized for $30-80 \mathrm{MHz}$, while the separate upper range (120$240 \mathrm{MHz}$, avoiding the FM radio broadcasting band) is covered with high-band antenna tiles (HBA) of $4 \times 4$ dipoles with built-in amplifiers and an analogue beam-former. Both receptor types are shown in Fig. 1.

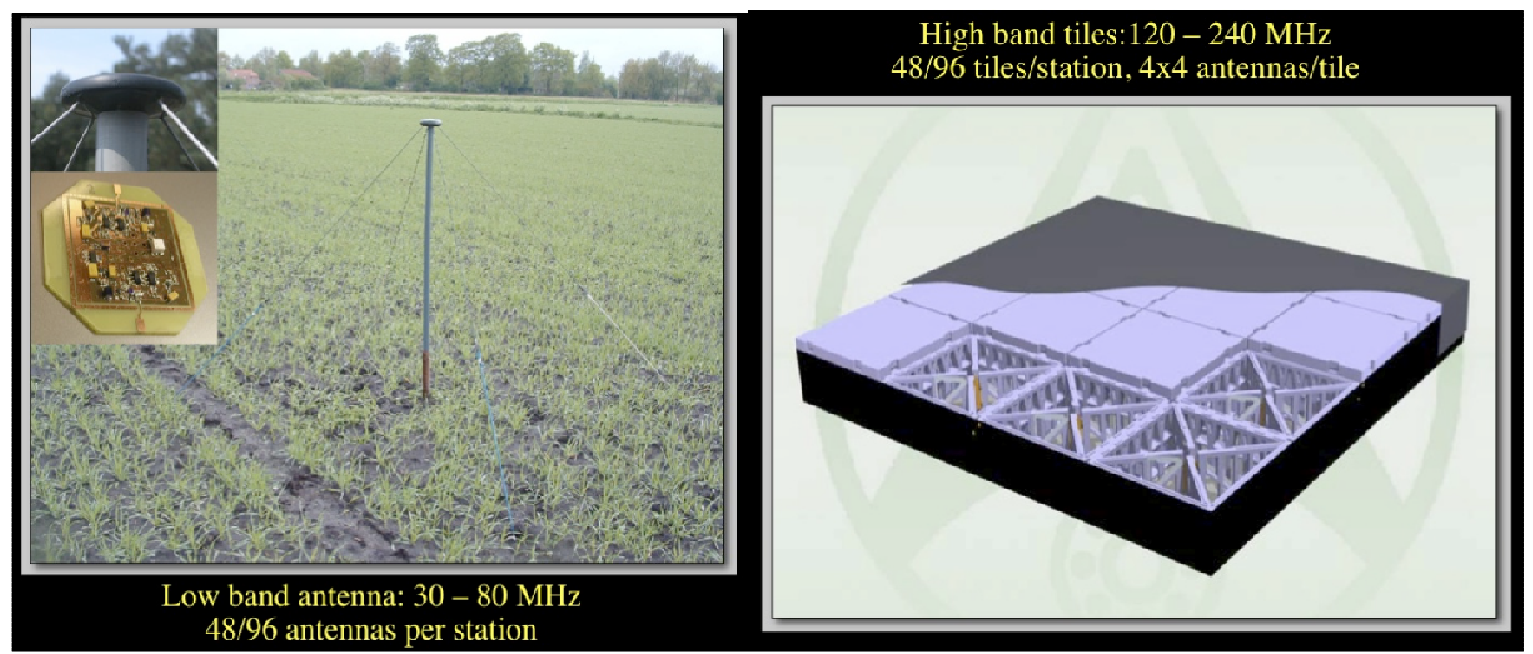

Figure 1. Low-band antenna dipoles (left) and high-band antenna tiles of dipoles (right) give very wide fieldof-view.

Each LOFAR station in the Netherlands has 96 LBA dipoles (selectable at any time as either 48 inner or 48 outer elements) and 48 HBA antenna tiles; international stations have both 96 LBA antennas and 96 HBA tiles. This architecture, with effectively all-sky coverage using many low-cost dipoles, gives LOFAR an unprecedentedly large field-of-view (many degrees, depending on frequency). The signals from individual dipoles or tiles are combined digitally into phased-array stations. Layered electronic beam-forming techniques at the tile, station, and multi-station levels not only allow for rapid re-pointing of the telescope but even for simultaneous observing of multiple, independent areas of the sky, as illustrated in Fig. 2. A unique feature of the LOFAR stations are the transient buffer boards, that are soon to be extended to allow the recovery at any moment of the last 5.2 seconds of full bandwidth unaveraged data from the individual dipoles or tiles. This allows post-facto beam-forming in any arbitrary direction, in response to an externally or internally generated trigger concerning a transient event anywhere on the sky. 


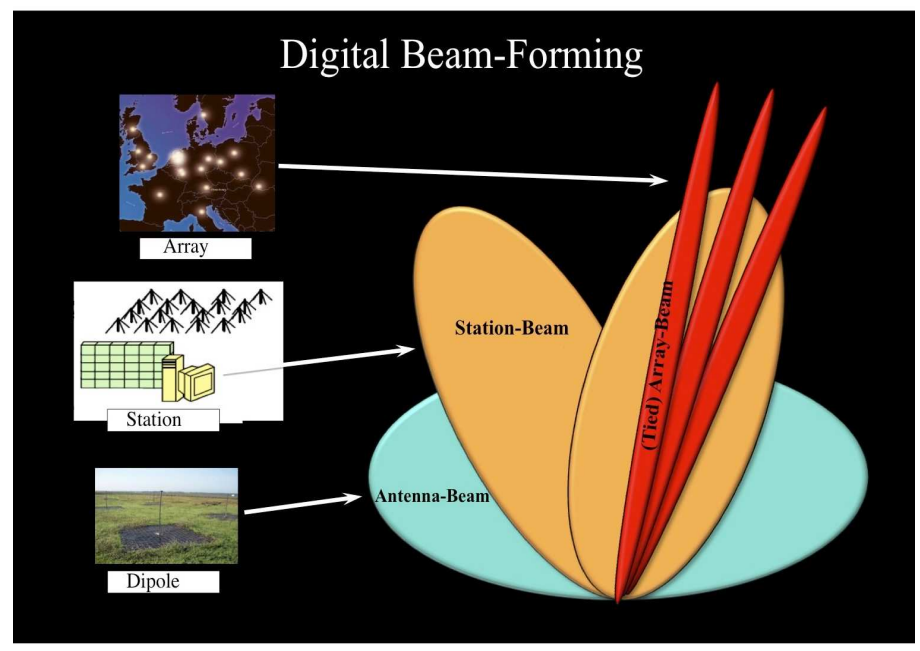

Figure 2. Electronic beam-forming at the station and multi-station levels allows the wide field- ofview of the individual LOFAR dipoles to be used for simultaneous observations of multiple, independent areas of the sky.

\subsection{Station and array topology}

The core area in the Netherlands was chosen to be near the town of Exloo in the northeastern province of Drenthe, which has relatively low population density and a comparatively benign radio frequency interference environment, which is reinforced through collaboration with a nature conservancy agency to establish a wetlands area. The distribution of the LOFAR core stations is optimized for high-fidelity imaging of spatially extended sources: there are 24 stations within the inner $2 \mathrm{~km}$ wide area, of which 6 are densely packed onto a $300 \mathrm{~m}$ diameter area called the Superterp, that is slightly raised above the surrounding wetlands. Most of the planned 16 remote stations out to distances up to $80 \mathrm{~km}$ in the Netherlands have now also been rolled out; these allow good higher resolution imaging. The array in the Netherlands is currently further complemented with 5 stations in Germany, and 1 each in France, Sweden, and the United Kingdom; these give baselines up to $1000 \mathrm{~km}$ and allow subarcsecond resolution in the high band. Further European stations are in advanced stages of planning. The international stations, while individually owned, are jointly operated within the ILT. The inner and outer topologies of the ILT are illustrated in Fig. 3.

\subsection{Central processing and storage}

All ILT stations can be centrally controlled from the operations room of ASTRON in Dwingeloo, the Netherlands. Each station can also be operated locally in stand-alone mode; some international station owners have installed local data processing equipment for private observations in addition to participating in ILT observing. All stations have dedicated (owned or leased) $3 \mathrm{~Gb} / \mathrm{s}$ or $10 \mathrm{~Gb} / \mathrm{s}$ data connections to the LOFAR central processing facilities at the computing centre of the University of Groningen in the Netherlands. The entire array, or arbitrary subsets of stations, can operate in interferometric mode for imaging. The core can be coherently or incoherently summed to give highly sensitive tied-array beams for surveying. Any and all single station data streams flowing into Groningen can also be used for time series or dynamic spectra. 
The data processing capacity in Groningen currently consists of a BlueGene/P supercomputer offering 42 Tflops and $640 \mathrm{Gbit} / \mathrm{s} \mathrm{I} / \mathrm{O}$, connected to a multi-node CPU cluster with 20 Tflops and 2 Pbyte temporary storage for post-processing operations. The ILT long-term archive is expected to be expanding at a rate of 2-4 Pbyte per year, based on collaborations involving the computing centers of Amsterdam, Groningen, and Jülich with other partners. While the raw visibility datasets are too large to store, the long-term archive will contain averaged visibilities as well as processed data such as images, time series, source and event lists, etc. The data in this e-archive will be open to the general public after a proprietary period (by default one year), and are expected to play an important part in the overall science yield obtained with this revolutionary new instrument.

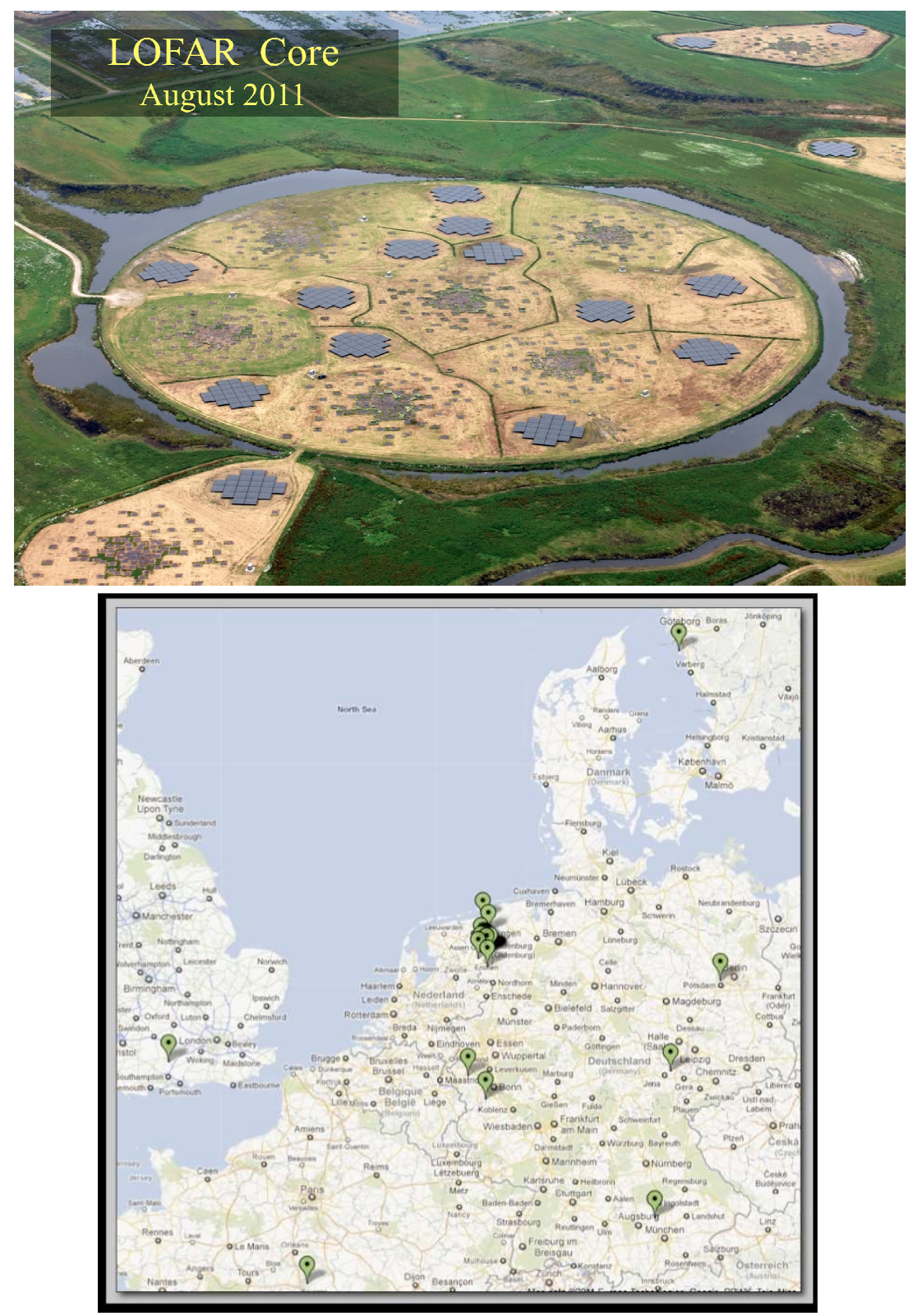

Figure 3. Top: The LOFAR Superterp is the innermost area, densely packed with the hardware of 6 stations. It is surrounded by several of the innermost core stations, visible as separate patches. Bottom: the locations of the current 8 international LOFAR stations in relation to the concentration of stations in the Netherlands. 


\section{The International LOFAR Telescope Foundation}

As part of the formal opening of LOFAR by Her Majesty queen Beatrix of the Netherlands, on 12 June 2010, a memorandum of understanding was signed by ASTRON and LOFAR astronomy consortia in Germany, France, the Netherlands, Sweden, and the United Kingdom. This paved the way to the formation in the Netherlands of the ILT Foundation, on 9 November 2010, as the vehicle for the international collaboration between the owners of LOFAR infrastructure (stations, computing, archives) and the astronomical stakeholders. The ILT seeks to maximize the science yield for its stakeholders and the full astronomical community, through the joint exploitation under a common science policy of the LOFAR infrastructure owned by the partners.

The LOFAR astronomical consortia bundle the interests of the parties involved in LOFAR (universities and other research institutes, station owners, etc.) in their country. The detailed setup of the consortia varies with local needs. ASTRON is the central operational organization within the ILT; all other station owners share in the cash and in-kind costs of central operations. Since 21 June 2011, the ILT Board, which sets the overall science policies, has representatives of ASTRON and all of the consortia in the countries where LOFAR stations are currently located.

\section{First proposal call with Open Skies}

After a period of concerted commissioning work, the ILT is now reviewing proposals in response to its first call for regular proposals. This call for the first time allows participation in LOFAR from the worldwide community. During Cycle 0, starting on 01 December 2012, $10 \%$ of the available operational time will be available under a fully Open Skies policy based purely on scientific merit as well as technical feasibility. After uniform review of all regular proposals, other parts of the observing time will be allocated in tandem between the independent Programme Committee and the individual LOFAR astronomy consortia listed above. They will generally also take into account relevance in their respective local contexts. This mechanism also allows a number of large Key Science Projects to get under way. The fully Open Skies time will expand later. A list of accepted proposals is kept publicly online.

For Cycle 0, there is a limited but diverse set of standard observing and data processing capabilities. LOFAR Version 1 delivers correlated visibility data, and in/coherently added single and multiple station data (several beam-formed modes), and transient buffer read-out. Synthesis images can be produced, and there is also a pipeline for known pulsar data processing. LOFAR Version 1 already allows novel and cutting-edge science projects, while considerable development is ongoing to reach the full potential of the ILT. Current limitations, such as on field sizes, resolutions, and noise levels of initial images if produced from an automatic pipeline, are explained online. Many science goals depend on further processing steps requiring expertise and/or specific tooling. Computing and storage for data processing will be a limiting resource for many projects; in this and many other respects, LOFAR is pioneering the regime in which the SKA will be operating. 


\section{Commissioning and first science results}

The past two years have been a period of intensive commissioning work, in which the software developers and astronomers at ASTRON have collaborated closely with the Key Science Project teams. Below, the main goals of each of these Key Science Project teams and some of the most promising first results are briefly presented. The joint commissioning work also extends to the Multi-Snapshot Sky Survey (MSSS), which is designed to yield an all-sky catalogue of calibrator sources, to be used for detailed calibration of the deeper imaging observations that are to follow. The MSSS catalog itself will also be used for population studies and other astrophysical applications. MSSS was started in late 2011; the first pass of observing, covering the $30-80 \mathrm{MHz}$ (LBA) band, is currently being completed, and HBA observing will follow. The catalog and images will be put into the public archive. The requirements of MSSS have been a prime driver both to develop the full, robust end-to-end LOFAR observing system, as well as to develop an initial automatic imaging pipeline, which is currently being extended and improved.

There are six Key Science Projects, each in fact covering a range of broad astrophysical topics that are enabled by a particular observing technique or feature of the LOFAR functionality. The design specifications and the development of specific hardware and software for LOFAR have been driven in part by the requirements of these Key Science Projects, worked out in long-standing collaborations between the design team at ASTRON and the astronomers involved.

Arguably the most challenging Key Science Project is aimed at the detection of the Epoch of Reionisation via its spectral and spatial radio emission/absorption signature in the redshifted HI $21 \mathrm{~cm}$ line. Brightness temperature steps of about $30 \mathrm{mK}$ are expected to occur over a range in redshift that overlaps the LOFAR HBA frequency range $(z=6-11)$. The team intends to observe a small number of fields for several hundreds of hours each. The cardinal issue facing them is not so much to accumulate raw sensitivity, but rather to achieve very high dynamic range and precise subtraction of numerous foreground components, which places stringent requirements on calibration and repeatability. It is most gratifying, therefore, that for a single 6-hour observing run the team has already been able to reach a thermal-noise limited image $(0.15 \mathrm{mJy} / \mathrm{beam})$ with a 600000:1 dynamic range, for their primary chosen field, and after a lot of dedicated processing. A recent summary of this project was given by Zaroubi et al. [1].

Another group is preparing for a three-tiered large-area sky survey in multiple LOFAR radio continuum bands. The main science goals of the surveys Key Science Project team are to study the formation and evolution of the first massive black holes, galaxies, and clusters of galaxies at redshifts $\mathrm{z}=6$ and higher, to chart in detail the grand era of star formation at redshifts $\mathrm{z} \sim 3$, and to make in depth studies of the gas in galaxy clusters at $\mathrm{z} \sim 1$. Their plans are described in detail by Röttgering et al. [2]. The commissioning efforts with the team at ASTRON to date have focused on understanding the challenges of extremely wide-field imaging, that requires handling of the nonplanar and non-isoplanatic effects, and understanding the detailed response function of the antennas, antenna tiles, and antenna stations. The fact that the brightest sources need to be removed from the data regardless of their separation on the sky forms the central field of interest. The effort to reach the ultimate, noise-limited image quality using procedures that can be automated is still proceeding; handling the ionospheric phase screen under current solar maximum conditions is a 
particularly difficult challenge. The range of images that have already been produced, however, is already impressive; a gallery of examples is in Fig. 4.

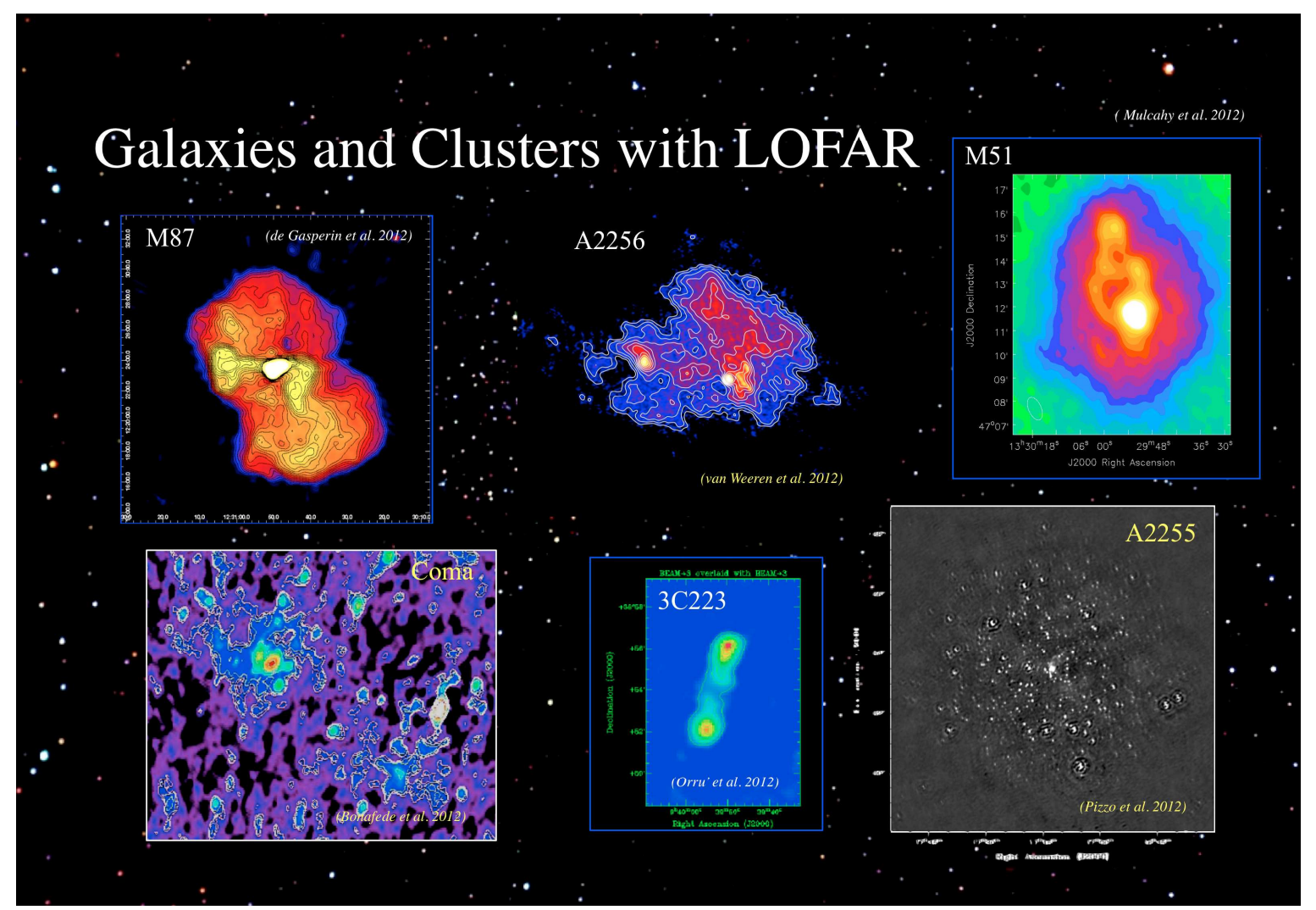

Figure 4. Gallery of early LOFAR images; some show the full field-of-view, others depict only the central source targeted by the observation. Credits to the main investigator involved are on each image.

There are also already impressive demonstrations of the use of the international LOFAR stations to achieve low frequency radio images with sub-arcsecond resolution, such as the pilot observation of Cas-A shown in Fig. 5.

A separate Key Science Project group focuses on achieving high quality full polarization imaging with LOFAR, in order to study the magnetic universe from near to far. Their main topics of interest include the origin of cosmic magnetism and the existence of seed fields and cosmic dynamos, magnetic collimation and acceleration models for mass flows, the physics of synchrotron radiation and interaction with particles in radio hot spots, and studies of planetary atmospheres. A recent overview of this project was given by Anderson et al. [3].

A further Key Science Project group is dedicated to solar and interplanetary medium studies. It will use both imaging and dynamic spectrum modes. Detailed goals have recently been described by Mann et al. [4]. Observing the Sun - a strong, variable, and extended source - is obviously rather more challenging than imaging a more typical astronomical field. Preliminary images of the Sun have already been produced and verified against images produced by other telescopes; this work is being extended to achieve routine snapshot solar imaging at higher resolution, as well as the production of dynamic spectra. 


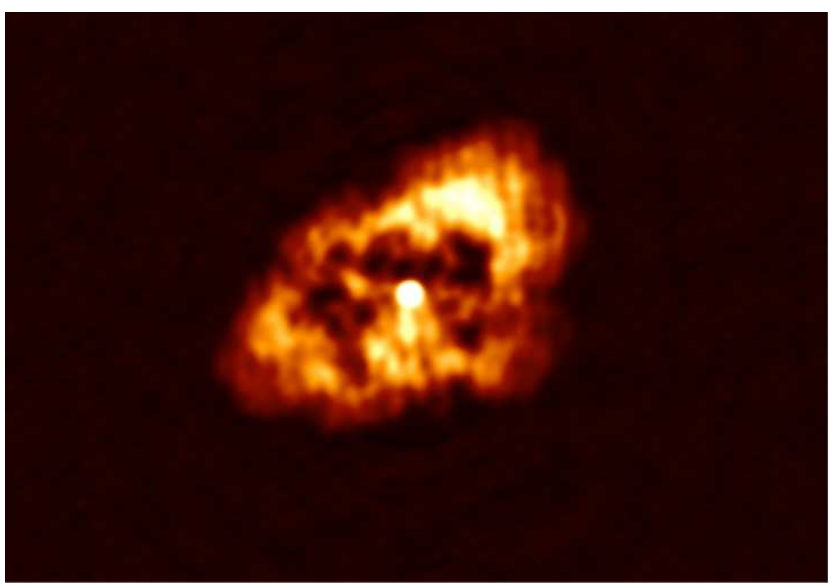

Figure 5. LOFAR high-band image of the Crab Nebula by Wucknitz et al.; in this complex field, the central pulsar is detected at sub-arcsecond resolution using the international LOFAR baselines.

LOFAR is also eminently suited for studies of variable sources, including exoplanets, variable stars and pulsars, as well as supermassive black holes and gamma-ray burst sources; see the recent summary by Fender [5]. Pulsar observations, both targeted to known pulsars, as well as surveys, have already started, based on the excellent sensitivity and high time resolution of LOFAR through its coherent beam-formed mode for the core stations, as described extensively by Stappers et al. [6]. Variable source surveys can already occur by analyzing series of images made at intervals ranging from months down to hours. This is being extended to snapshots in a cadence as rapid as minutes and even seconds. Software also is being developed to allow patrols for variable sources to occur in parallel on the data for any targeted imaging observation. Transient studies will also benefit tremendously from LOFAR's unique transient buffer boards. These revolving buffers can hold the last 5 seconds of data, and longer at reduced bandwidth. Upon receipt of an internally or externally generated trigger, and interruption of the running observation, this data can be read back to form an a posteriori image anywhere in the field of the individual dipole antennas or tiles; in the low frequency band, this means most of the instantaneously visible sky!

The LOFAR transient buffer boards are also essential for the final Key Science Project, on the detection of high-energy cosmic rays. As described and demonstrated by Corstanje et al. [7], such particles lead to air showers when they impact the upper atmosphere, of which the short flash of radio emission can be detected by LOFAR, with the precise timing, spectrum, and footprint giving both localization as well as a measure of the energy of the particle. An even more ambitious project is in preparation, that aims to detect radio flashes from ultra-high energy cosmic rays impacting the surface of the Moon.

In short, with the first call for regular proposals, heralding the start of both the Key Science Projects as well as the Open Skies access, that should draw both new expertise and new ideas from the wider community, LOFAR is poised to make a major impact in many areas of astronomy, with revolutionary observing capabilities that will be steadily expanding in the next few years.

\section{Acknowledgements}

This paper is closely based on earlier symposium papers by the same author [8,9], and represents part of the efforts of the full LOFAR collaboration. 


\section{References}

[1] Zaroubi, S., de Bruyn, A. G., Harker, G., et al. 2012, MNRAS, 425, 2964

[2] Röttgering, H., van Weeren, R. J., Miley, G., et al. 2010, Proceedings of the International SKA Forum 2010, PoS (ISKAF2010) 050

[3] Anderson, J., Beck, R., Bell, M., et al. 2012, Proceedings of the conference Magnetic Fields in the Universe: From Laboratory and Stars to Primordial Structures, Eds. M. Soida, K. OtmianowskaMazur, E. M. de Gouveia Dal Pino, and A. Lazarian, arXiv:1203.2467

[4] Mann, G., Vocks, C., \& Breitling, F. 2011, Proceedings of the 7th International Workshop on Planetary, Solar and Heliospheric Radio Emissions, Austrian Academy of Sciences Press, p. 507.

[5] Fender, R. 2012, Proceedings of the IAU Symposium 285, New Horizons in Time-Domain Astronomy, Eds. R. E. M. Griffin, R. J. Hanisch, and R. Seaman, p. 11

[6] Stappers, B. W., Hessels, J. W. T., Alexov, A., et al. 2011, A\&A, 530, A80

[7] Corstanje, A., van den Akker, M., Bähren, L., et al. 2011, Proceedings of the 32nd International Cosmic Ray Conference, arXiv:1109.5805

[8] Vermeulen, R. C. 2012, Proceedings of the conference Resolving the Sky - Radio Interferometry: Past, Present and Future, PoS (RTS2012) 035

[9] Vermeulen, R. C. 2012, Proceedings of the SPIE, Ground-based and Airborne Telescopes IV, Vol. 8444, id. 84442B 